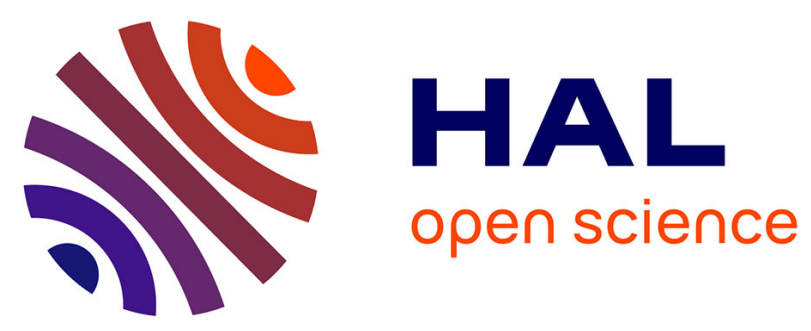

\title{
Appréciation de l'état hydrique d'une plante à partir des variations micrométriques de la dimension des fruits ou des tiges au cours de la journée
}

\author{
Jean-Gérard Huguet, Bernard Jaussely, Patrick Orlando
}

\section{- To cite this version:}

Jean-Gérard Huguet, Bernard Jaussely, Patrick Orlando. Appréciation de l'état hydrique d'une plante à partir des variations micrométriques de la dimension des fruits ou des tiges au cours de la journée. Agronomie, 1985, 5 (8), pp.733-741. hal-00884806

\author{
HAL Id: hal-00884806 \\ https://hal.science/hal-00884806
}

Submitted on 1 Jan 1985

HAL is a multi-disciplinary open access archive for the deposit and dissemination of scientific research documents, whether they are published or not. The documents may come from teaching and research institutions in France or abroad, or from public or private research centers.
L'archive ouverte pluridisciplinaire HAL, est destinée au dépôt et à la diffusion de documents scientifiques de niveau recherche, publiés ou non, émanant des établissements d'enseignement et de recherche français ou étrangers, des laboratoires publics ou privés. 


\title{
Appréciation de l'état hydrique d'une plante à partir des variations micrométriques de la dimension des fruits ou des tiges au cours de la journée
}

\author{
Jean-Gérard HUGUET \\ avec la collaboration technique de Bernard JAUSSELY \& Patrick ORLANDO
}

I.N.R.A., Station d'Agronomie, Centre de Recherches Agronomiques d'Avignon, F 84140 Montfavet

La mesure, non destructive, à l'échelle du micromètre $\left(10^{-3} \mathrm{~mm}\right)$ des variations de dimensions des tiges ou fruits d'un végétal dans son milieu naturel révélerait l'intensité de la sollicitation de ses propres réserves hydriques, réserves capables de pallier, jusqu'à un certain point, un déficit de son bilan hydrique. Le dépassement d'une limite, amplitude critique de contraction diurne, pourrait signaler l'insuffisance de la disponibilité de l'eau dans le sol, donc un besoin d'irrigation. L'avantage de ce procédé serait d'autoriser une programmation de l'irrigation avant que le manque d'eau n'ait perturbé le fonctionnement de la culture.

Mots clés additionnels : Réserves hydriques, transpiration végétale, automatisation de l'irrigation, déficit hydrique, pommier, citronnier.

Measurements of diurnal micrometric contractions and expansions were made on several plants under natural conditions. The results were assumed to be related to variation in the amount of the water stored in the plant itself. A water balance deficit may, to some extent, be overcome by this stored water. Measurement of a boundary value of a diurnal contraction could be chosen as a good indicator of the need for irrigation. Such a device could be used to programme irrigation and prevent water stress from ever occuring.

Additional key words : Water storage, plant transpiration, irrigation, irrigation automation, water stress, apple tree, citrus.

\section{INTRODUCTION}

L'ambition générale des agronomes est d'optimiser l'emploi des facteurs de production (eau, éléments minéraux, énergie, etc...) dans le souci d'une économie des moyens et d'une réduction des nuisances.

La problématique concernant l'étude de la physiologie d'un être vivant conduit généralement à contrôler les flux de matières et d'énergie dont il est le siège såchant que l'état de vie active ne supporte pas longtemps un bilan négatif. Tout indicateur qualitatif (sens) ou quantitatif (intensité) de flux est précieux, voire indispensable dans l'approche du vivant. Mais les flux sont des phénomènes dynamiques dont l'étude doit respecter l'intégralité fonctionnelle du système concerné pour avoir une signification utile.

Expérimentalement, et quelle que soit la méthodolo- gie appliquée, l'accès au flux peut être obtenu par une succession d' " instantanés » destructifs échelonnés dans le temps sur des individus différents ou par des mesures en continu sur le même individu, la seconde approche étant naturellement beaucoup plus « économe " vis-à-vis des précautions statistiques liées à la prise en compte des variations individuelles.

Dans une première étape, un suivi micrométrique des variations de dimensions des différents organes d'une plante paraissait une voie prometteuse pour une perception globale de ses échanges hydriques.

Depuis longtemps, le suivi à l'échelle du $\mu \mathrm{m}$ $\left(10^{-3} \mathrm{~mm}\right)$ des variations de dimensions d'organes ligneux (tronc, tige, rameaux) ou de fruits, à un pas de temps court, a montré des fluctuations périodiques dont une partie pouvait être mise en relation avec l'alimentation hydrique de la plante. 
Le phénomène essentiel mis en évidence, en pleine saison végétative, est une phase de réduction de diamètre, qui, par une journée ensoleillée, s'amorce en début de matinée pour se terminer dans le courant de l'après-midi avec une amplitude totale de quelques centaines de micromètres (BARTHOLOMEW, 1923: citrus ; HALMA, 1934 : citrus ; SCHROEDER \& WIEDLAND, 1956 : avocatier). Ce dernier auteur montre, sur fruit (avocat), qu'après le minimum atteint dans l'après-midi, une phase de croissance régulière s'installe avec une pente telle que le fruit retrouve dans la nuit sa dimension maximum du jour précédent puis continue à croître en diamètre de quelques centaines de $\mu \mathrm{m}$ pour atteindre un nouveau maximum avant une nouvelle phase de décroissance. La courbe des variations de diamètre d'un fruit prend une allure typique en dents de scie (cf. fig. 2) avec une tendance globale à la croissance interrompue par des épisodes quotidiens de décroissance.

Cet auteur prouve que la demande hydrique climatique est à l'origine de la diminution au cours de la journée du diamètre des fruits qui fournissent de l'eau (5 à 6 p. 100 de leur poids total) au profit de la transpiration des feuilles. Ces mouvements d'eau à partir des fruits vers les feuilles avaient déjà été prouvés (HODGSON, 1917 : citrus ; BARTHOLOMEW, 1923) et font partie du domaine de l'observation courante : en cas de sécheresse, un arbre sans fruit flétrit avant un arbre chargé de fruits. Des mesures sur organes ligneux, tronc, tige, racine montrent au cours de la journée une diminution d'amplitude analogue à celle observée sur fruit (SCHROEDER \& WIEDLAND, 1956 : avocatier ; LASSOIE, 1973 : arbres forestiers). En 1971, CHANEY \& KOZlOWSKI confirment sur les fruits d'un citrus nain l'ensemble de ces résultats qui sont également retrouvés sur tige de cotonnier (MOLz \& KLEPPER, 1972) et sur fruit et tronc de pommier (POWELl \& THORPE, 1975) à l'occasion de tentative de modélisation de ces variations de dimension à partir de mesures des potentiels hydriques à la bombe de SCHOLANDER et al. (1965). D'une façon générale, ces 10 dernières années, la théorisation du transfert d'eau à travers le système " sol-plante-atmosphère " a montré la nécessité de prendre en compte les « réservoirsinternes " de végétaux (cf. par ex. KATERJI, 1982).

L'objectif de ce travail est une tentative d'analyse de la signification des microvariations nycthémérales dans les dimensions de certaines parties d'une plante vivante. Une attention particulière est portée à la signification de ces microvariations par rapport à l'état hydrique de la plante.

\section{MATÉRIELS ET MÉTHODES}

\section{A. Type de capteur}

Sur les 30 dernières années, la quasi-totalité des équipes qui ont utilisé les mesures micrométriques ont choisi des capteurs de déplacement linéaire à induction différentielle. Nous avons fait le même choix en raison du degré de perfection remarquable des modèles actuels (fiabilité, légèreté, tenue à l'humidité, miniaturisation, résolution): Capteurs de déplacements rectilignes Enertec-Schlumberger ; à courant alternatif série $C D 20$; à courant continu série CD 40 .

\section{B. Porte-capteurs (fig. 1 ; photo 1)}

La difficulté essentielle de l'emploi d'un capteur à haute résolution $(\simeq 1 \mu \mathrm{m})$ dans les conditions $d u$ plein champ est de réussir à ce que le corps du capteur et l'organe à mesurer soient maintenus dans un bâti de référence indéformable. A l'extérieur, il faut supprimer les mouvements parasites d'origine mécanique (vent, choc...) et les dilatations dues aux variations de températures ; les effets thermiques ont été éliminés en utilisant de l'INVAR (alliage Fe Ni à coefficient de dilatation pratiquement nul) pour les 2 modèles de bâti de référence.

Quant aux effets mécaniques, ils ont été pratiquement supprimés par la géométrie des porte-capteurs adaptés aux fruits (carpomètre) ou aux tiges (dendromètre) et la position des pièces de maintien de telle façon que ces bâtis restent solidaires de l'organe à mesurer quels que soient ses mouvements, qui restent libres. La seule précaution sur fruit est de soulager le pédoncule du poids de l'ensemble de mesure (environ $180 \mathrm{~g}$ pour le carpomètre) en suspendant ce dernier à une autre partie de l'arbre. Il est également indispensable de coller la tige sensible à l'objet (toile adhésive double face ou bien colle tolérée par l'épiderme). Dans la mise en place des carpomètres, un certain " doigté » est nécessaire pour manipuler le fruit avec délicatesse et régler la pression des ressorts de stabilisation sans contrainte excessive. Suivant l'amplitude de mesure des capteurs ( $5 \mathrm{~mm}$ dans notre cas) et la croissance nette des fruits d'un jour à l'autre, ces réglages sont à corriger tous les 10 à $15 \mathrm{j}$.

\section{Saisie et traitement des données}

\section{Deux systèmes sont utilisés :}

- Le 1 ${ }^{\text {er }}$, organisé autour d'une centrale de saisie de 12 voies qui gère le temps, recueille les données analogiques et les transmet sous forme digitale à un microcalculateur pour l'ensemble des traitements informatiques (calculs, stockage données, sorties graphiques, visualisation temps réel, etc...). Ce dispositif est adapté à des expériences situées à moins de $100 \mathrm{~m}$ du bâtiment.

- Pour surmonter cet aspect limitant, le $2^{\mathbf{e}}$ système totalement autonome est organisé autour d'un microcalculateur à mémoire permanente (Microcalculateur " Husky » 8 entrées analogiques $( \pm 4 \mathrm{~V})$, doté d'une entrée avec convertisseur analogique digital pour 8 voies. L'autonomie d'alimentation électrique de ce dispositif autorise des mesures sur n'importe quel site; lorsque la période de mesure est terminée, le microcalculateur, ramené au laboratoire, décharge ses mémoires sur un autre calculateur ou sur des périphériques adaptés à l'utilisation souhaitée.

\section{Matériel végétal}

La souplesse d'emploi des systèmes de mesure utilisés a permis de suivre du matériel végétal varié (pommier, citronnier, maïs, tournesol) en pleine terre avec plusieurs systèmes d'irrigation, ou en pot (citronnier) de façon à bien maîtriser la disponibilité de l'eau. 


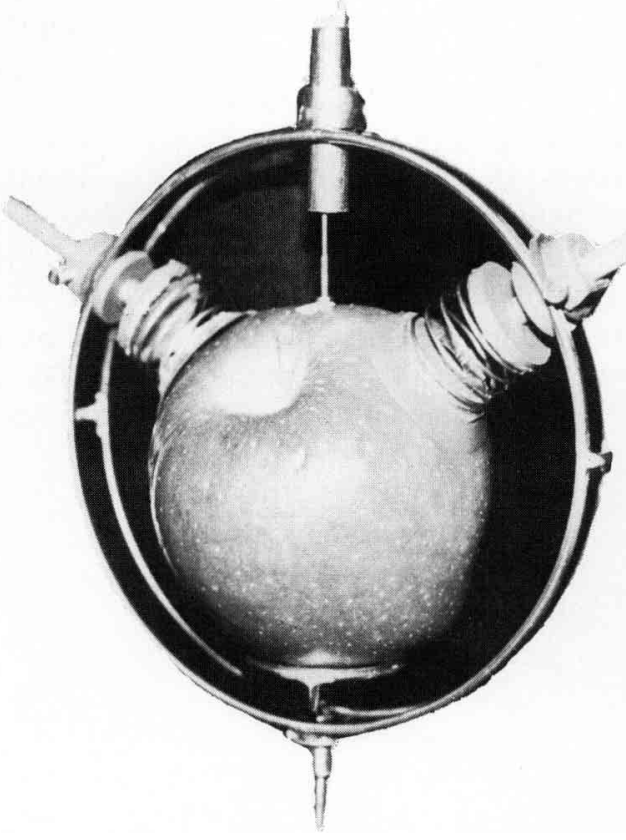

Photo 1

Carpomètre en place sur une pomme.

Carpometer on a fruit.

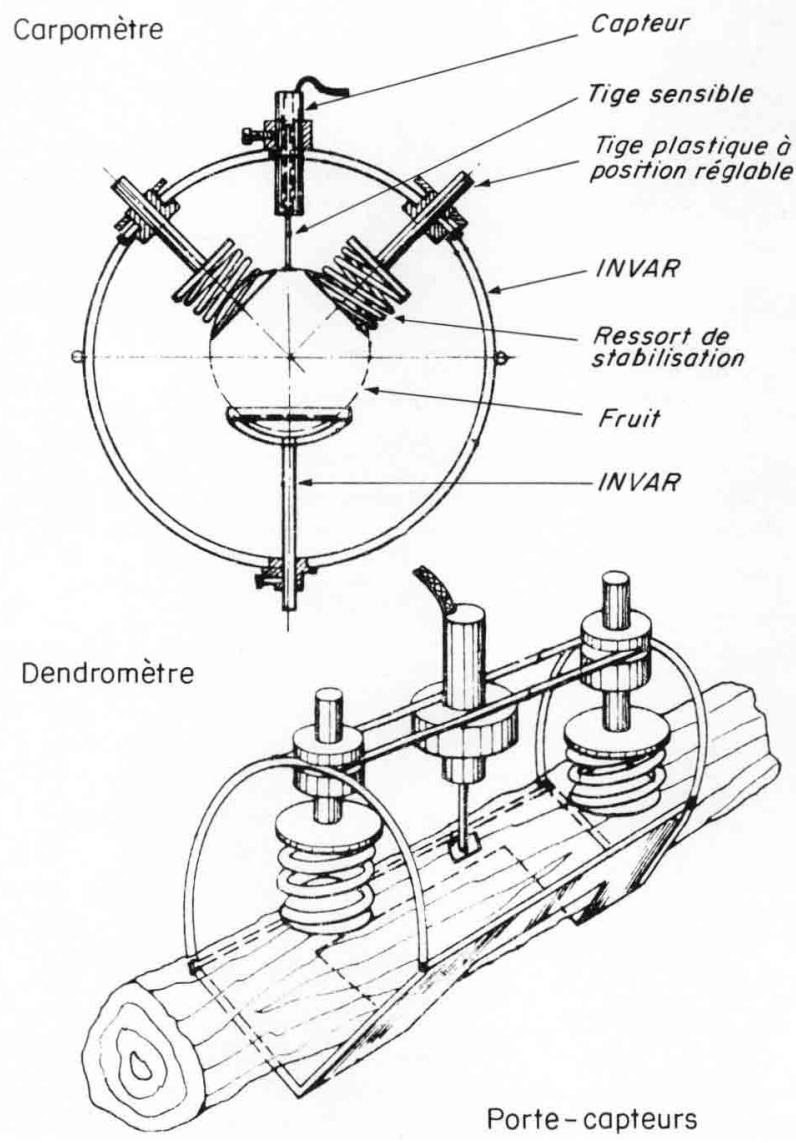

Figure 1

Porte-capteurs de mesure des microvariations de diamètre.

Gauge holder for micrometric measurements on fruit (carpometer) and stem (dendrometer).

\section{Figure 2}

Fluctuation saisonnière des contractions sur pommes. Seasonal variation in the contractions of apple fruits.

\section{RÉSULTATS}

\section{A. Allure générale des variations de dimensions}

La figure 2 montre l'évolution des diamètres de pommes ( Golden delicious ») en sol d'alluvions (limono-argileux) bien irrigué au cours de 2 périodes climatiques très différentes:

- Période caniculaire (maximum supérieur à $40{ }^{\circ} \mathrm{C}$ ) en juillet : l'aspect classique de croissance en « dents de scie » est bien apparent avec une tendance générale à la croissance du fruit lié au transfert d'assimilats vers le fruit et une décroissance diurne très nette en $8 \mathrm{~h}$ et $18 \mathrm{~h}$.

- Un mois plus tard, au cours d'une période moins chaude (maximum $30^{\circ} \mathrm{C}$ ) : la croissance générale est plus faible, la contraction diurne plus tardive, son amplitude est très faible alors que la contraction d'une charpentière est très importante (fig. 3 , période homologue : août 1984).

Cette figure 3 montre que les contractions diurnes apparaissent au cours de journées chaudes et ensoleillées (les 3 premières journées) et que l'orage $(40 \mathrm{~mm}$ ) du 23 août avec un temps humide, frais et couvert supprime pratiquement ces contractions au cours des 2 derniers jours.

Les phénomènes de contractions et de dilatations qui affectent fruits et tiges ont une périodicité de $24 \mathrm{~h}$; leur amplitude varie apparemment en relation avec les circonstances climatiques. Ils se superposent, dans le cas des pommes, avec une tendance régulière à la croissance : grossissement cellulaire lié à l'afflux d'eau et aux transferts d'assimilats vers les fruits, alors que les organes ligneux (pommiers) sont à cette époque en pause de croissance secondaire.

\section{B. Rôle de la transpiration foliaire}

La figure 4 montre, sur une courte période de temps, l'évolution des dimensions de 2 citrons d'un même arbre dans un pot $(25$ l) bien arrosé et placé
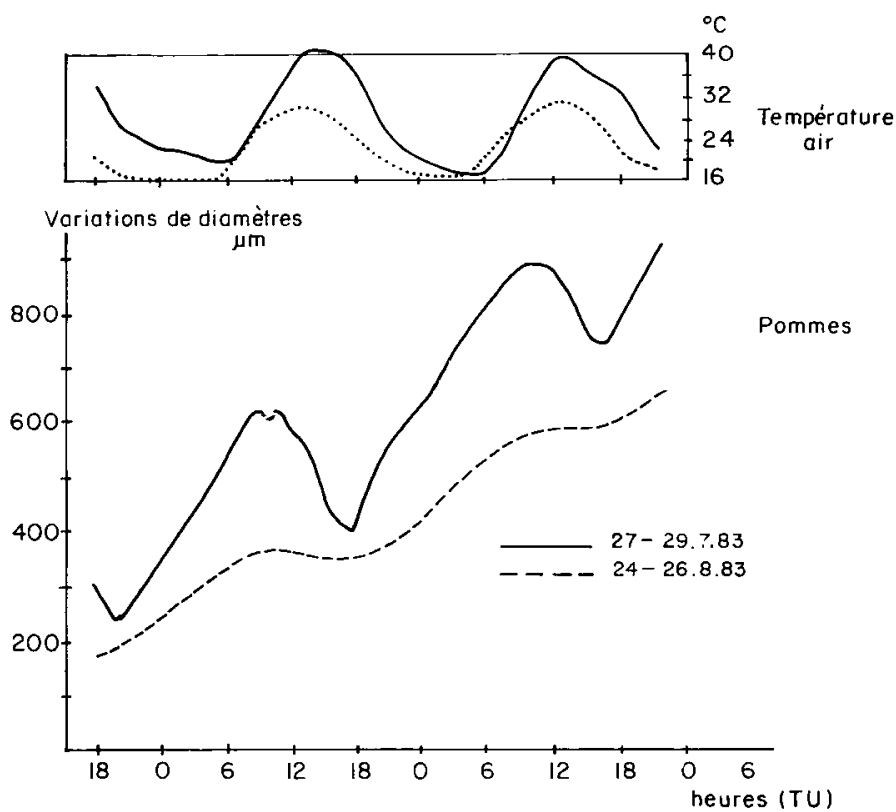
alternativement à l'obscurité et à la lumière indirecte, par occultation des fenêtres de la pièce dont la température est restée pratiquement constante pendant la durée de l'expérience $\left(25^{\circ} \mathrm{C}\right)$. Sur le citron 1 (encore vert), il est apparent que la contraction est déclenchée par la lumière, après un temps de latence d'une dizaine de minutes. Le citron 2 (jaune et mûr), bien qu'il soit encore bien fixé à son pétiole, peut être considéré comme séparé du reste de la plante par une couche d'abscission isolante; sa diminution régulière résulte de son évaporation propre, tout à fait négligeable par rapport à la contraction du citron 1 . Le phé- nomène à l'origine de la périodicité des contractions est donc probablement l'ouverture stomatique réglée par l'intensité lumineuse ; en phase de transpiration intense des feuilles, l'eau passe des fruits vers ces feuilles, l'amplitude de la contraction dépendrait, entre autres, des valeurs relatives de la demande climatique (intensité d'évaporation foliaire) et de la disponibilité de l'eau dans le sol (intensité de l'absorption racinaire).

Fruits et organes ligneux peuvent jouer, on le sait, le rôle de fournisseurs transitoires d'eau. Dans notre cas, ce rôle apparaît au cours des périodes diurnes de

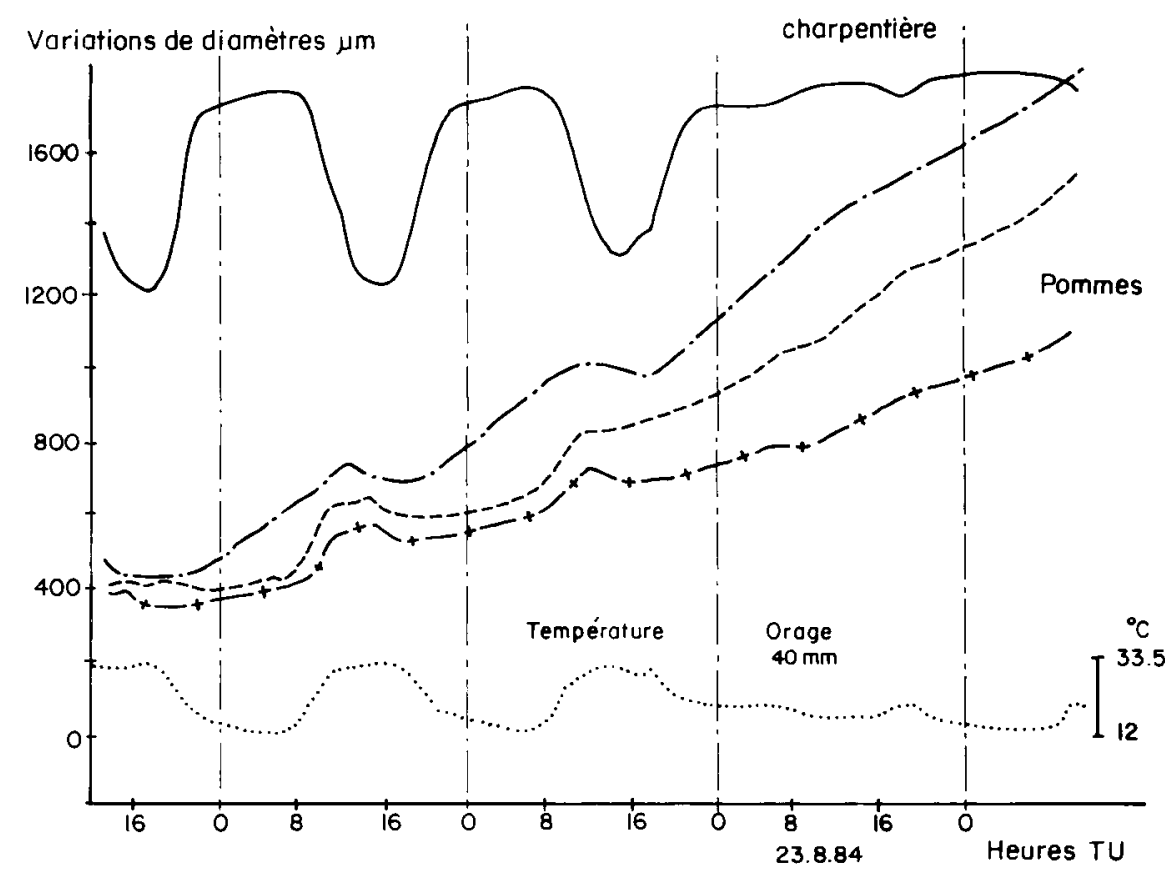

Figure 3

Contractions de la tige et des fruits d'un même arbre (pommier Golden).

Fruit and stem contractions on the same apple tree, with rainfall on 23 August 84.

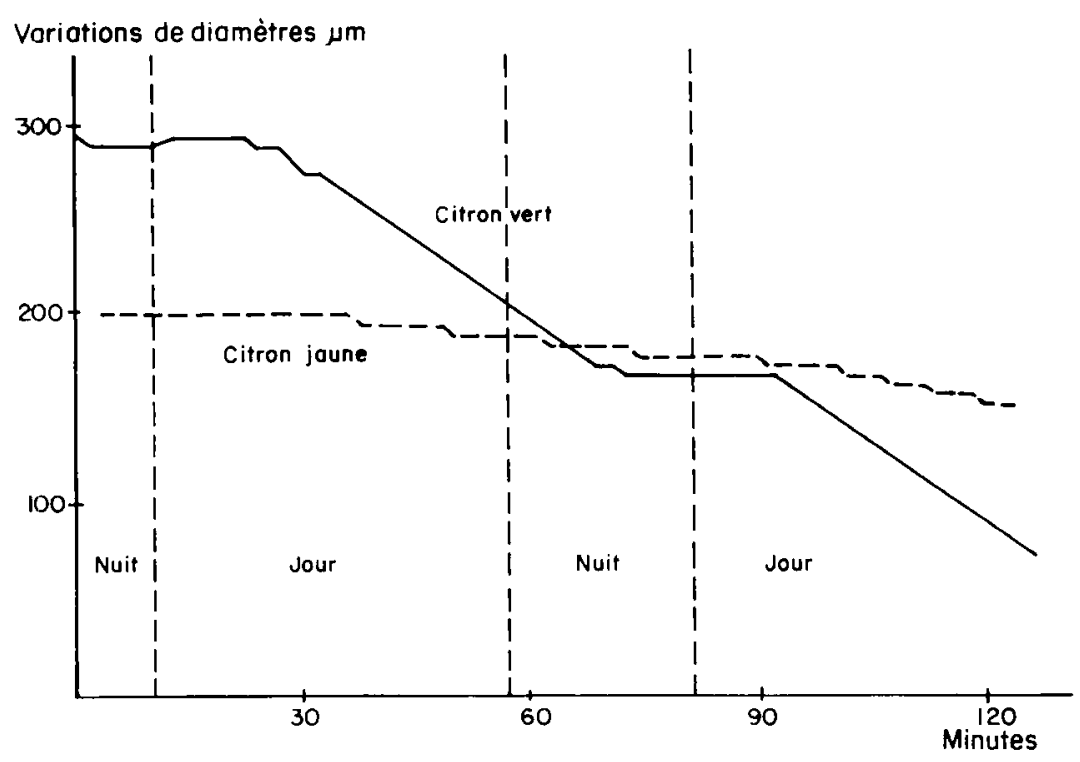




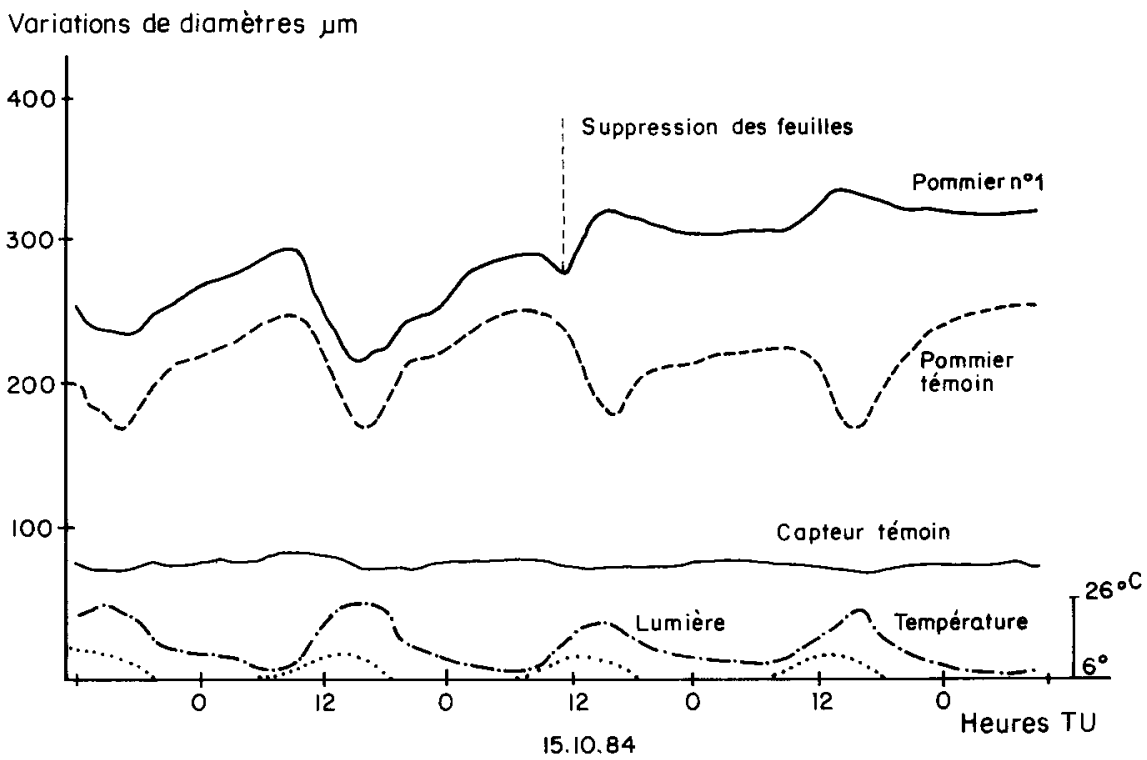

Figure 5

Effets de la transpiration foliaire sur les contractions de charpentières (pommier Golden).
Effects of leaf transpiration on the contraction of apple stems. forte demande climatique. Cette hypothèse est appuyée par les résultats d'une défoliation complète et artificielle (fig. 5) réalisée à $10 \mathrm{~h}$ du matin sur un pommier dont une charpentière amorçait sa contraction diurne. L'effet de cette défoliation déphase complètement cet arbre par rapport au témoin : son tronc se dilate (bilan hydrique positif) par reconstitution de ses réserves propres (tissus corticaux), accentué par un léger effet de dilatation thermique (cf. $\S \mathrm{D}$ ).

L'examen de la figure 6 apporte une autre illustration de la mise en jeu de ces réservoirs. Il s'agit de résultats obtenus sur un petit citronnier cultivé dans un pot de 101 installé sur une balance afin de suivre les variations de poids correspondant à la transpiration de l'arbre (surface du sol recouverte d'une feuille plastique). Pendant la $2^{\mathrm{e}}$ journée, la croissance du fruit marque un simple ralentissement; au cours de la $3^{\mathrm{e}}$ journée, la sollicitation des réserves d'eau du fruit est importante. Un apport de $100 \mathrm{ml}$ d'eau à $15 \mathrm{~h}$ permet une récupération partielle qui indique que le sol, en voie d'assèchement, ne fournit plus assez d'eau. Pendant la $4^{\mathrm{e}}$ journée, la sollicitation intense des réserves hydriques du fruit est à peine atténuée par un apport d'eau de $100 \mathrm{ml}$, le sol très sec retenant fortement la plus grande partie de l'eau apportée. Un apport plus important d'eau à $16 \mathrm{~h}(500 \mathrm{ml})$ au moment de la baisse de luminosité (expérience en serre en décembre) permet une remontée très rapide du diamètre du fruit qui regarnit ses réserves.

L'efficacité photosynthétique de l'arbre ne paraît pảs avoir diminué puisque le dernier maximum se retrouve sur la droite de grossissement moyen; de plus, au cours de ces $5 \mathrm{j}$, la transpiration moyenne a été très régulière. Il n'y a apparemment pas eu de régulation stomatique induite par la sécheresse du sol ; la participation des réserves hydriques de l'arbre a permis de tamponner les irrégularités de fourniture du sol.

\section{Origine et disponibilité des réserves internes}

La littérature (MOLZ \& KLEPPER, 1972) et nos propres mesures montrent que les contractions et dilatations d'une tige viennent essentiellement des variations d'épaisseur des tissus corticaux ; le bois (xylème) n'a pratiquement pas de variation de diamètre.

L'examen, sur un même arbre, des fluctuations micrométriques sur des tiges de différents diamètres

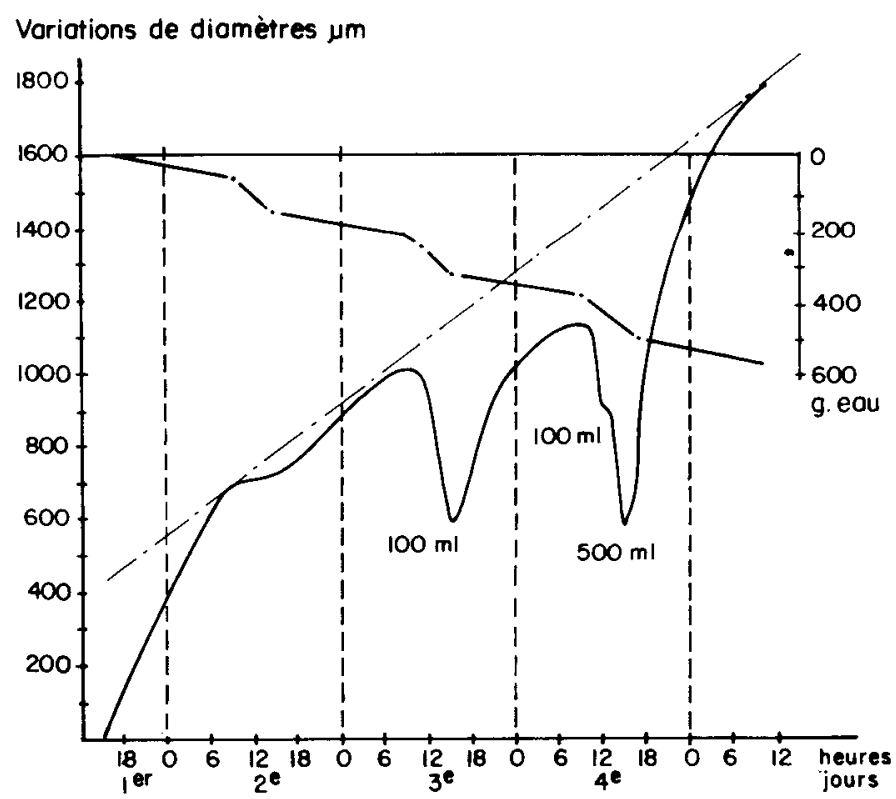

Figure 6

Evolution du diamètre d'un citron et de la consommation cumulée d'eau d'un arbre en pot.

Variation in the diameter of lemon fruits in relation to accumulated water consumption of a potted tree. 


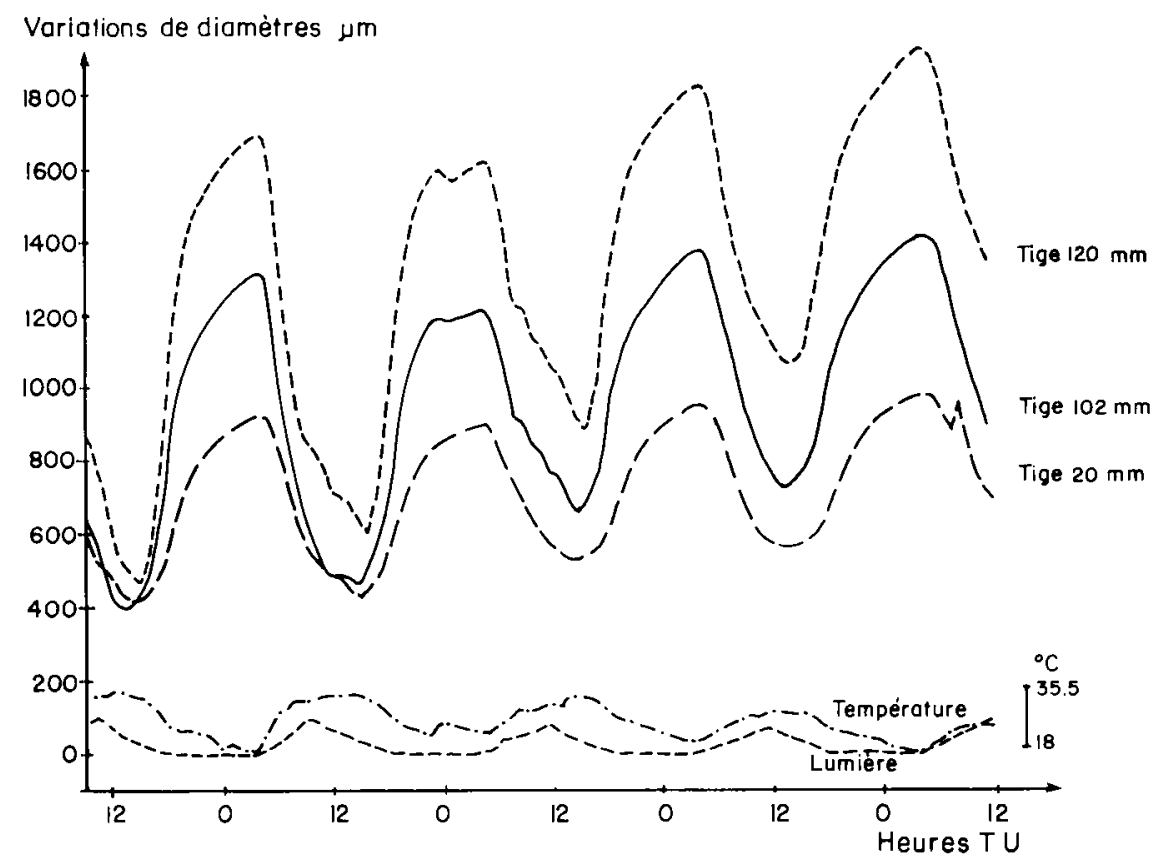

Figure 7

Contractions des tiges (pommier Golden) suivant leur diamètre (23 au 27.7.84).

(fig. 7) montre que l'amplitude absolue des contractions diurnes est positivement corrélée au diamètre de la tige. Mais comme l'épaisseur des tissus corticaux est également fonction du diamètre de la tige (fig. 8), il est possible d'exprimer les contractions diurnes en pourcentages de l'épaisseur corticale (tabl. 1).

Ces contractions relatives sont systématiquement plus fortes pour la tige de faible diamètre. Ce résultat pourrait suggérer l'hypothèse chez les ligneux d'une sollicitation d'autant plus forte des réserves hydriques que l'on est plus proche des feuilles.

La sollicitation des réserves varie sous l'effet du gradient de potentiel hydrique qui admet les feuilles comme point le plus bas dès que la luminosité induit une ouverture stomatique suffisante. En suivant avec un pas de temps de $30 \mathrm{mn}$ la vitesse des variations de dimension (contraction ou expansion) en relation avec celle de la transpiration (pesée d'un arbre en pot), il apparaît (fig. 10), par journées claires $\left(3^{\mathrm{e}}, 4^{\mathrm{e}}, 5^{\mathrm{e}}\right)$, que la contraction s'installe en début de matinée et devient
Stem contractions of the same apple tree in relation to diameter.

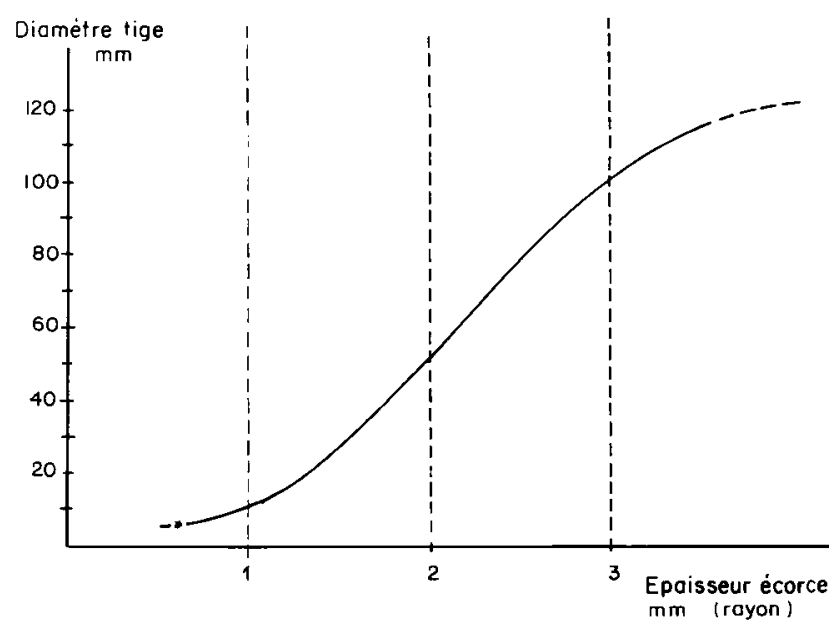

Figure 8

Epaisseur moyenne de l'écorce suivant le diamètre de la tige (pommier Golden).

Mean thickness of apple bark in relation to stem diameter.

TABLEAU

Contractions absolues de tige de pommiers suivant leurs diamètres et contractions relatives à l'épaisseur totale de l'écorce. Absolute stems contractions of apple in relation to diameter and contractions relative to total bark thickness.

\begin{tabular}{|c|c|c|c|c|c|c|c|}
\hline & & \multicolumn{6}{|c|}{ Juillet 1984} \\
\hline & & \multicolumn{2}{|c|}{24} & \multicolumn{2}{|c|}{25} & \multicolumn{2}{|c|}{26} \\
\hline & & $\mathrm{T}^{\circ} 30^{\circ} \mathrm{C}$ & Lum. 205 & $\mathrm{~T}^{\circ} 28,7^{\circ} \mathrm{C}$ & Lum. 182 & $\mathrm{~T}^{\circ} 26^{\circ} \mathrm{C}$ & Lum. 158 \\
\hline \multirow{2}{*}{$\begin{array}{c}\text { Diamètre } \\
\text { branche } \\
\text { mm }\end{array}$} & \multirow{2}{*}{$\begin{array}{c}\text { Total } \\
\text { écorce } \\
\text { mm }\end{array}$} & \multicolumn{2}{|c|}{ Contraction maximum } & \multicolumn{2}{|c|}{ Contraction maximum } & \multicolumn{2}{|c|}{ Contraction maximum } \\
\hline & & Abs. $\mu \mathrm{m}$ & Rel. \% Eco & Abs. $\mu \mathrm{m}$ & Rel. \% Eco & Abs. $\mu \mathrm{m}$ & Rel. $\%$ Eco \\
\hline 20 & 2,6 & 249 & 9,2 & 189 & 7,3 & 200 & 7,7 \\
\hline 102 & 6 & 431 & 7,2 & 285 & 4,7 & 325 & 5,4 \\
\hline 120 & 7,2 & 550 & 7,6 & 371 & 5,2 & 383 & 5,3 \\
\hline
\end{tabular}

$\left.\begin{array}{l}\mathrm{T}^{\circ}: \text { Température moyenne } \\ \text { Lum : Index moyen de lumière }\end{array}\right\}$ pendant la phase de contraction. 


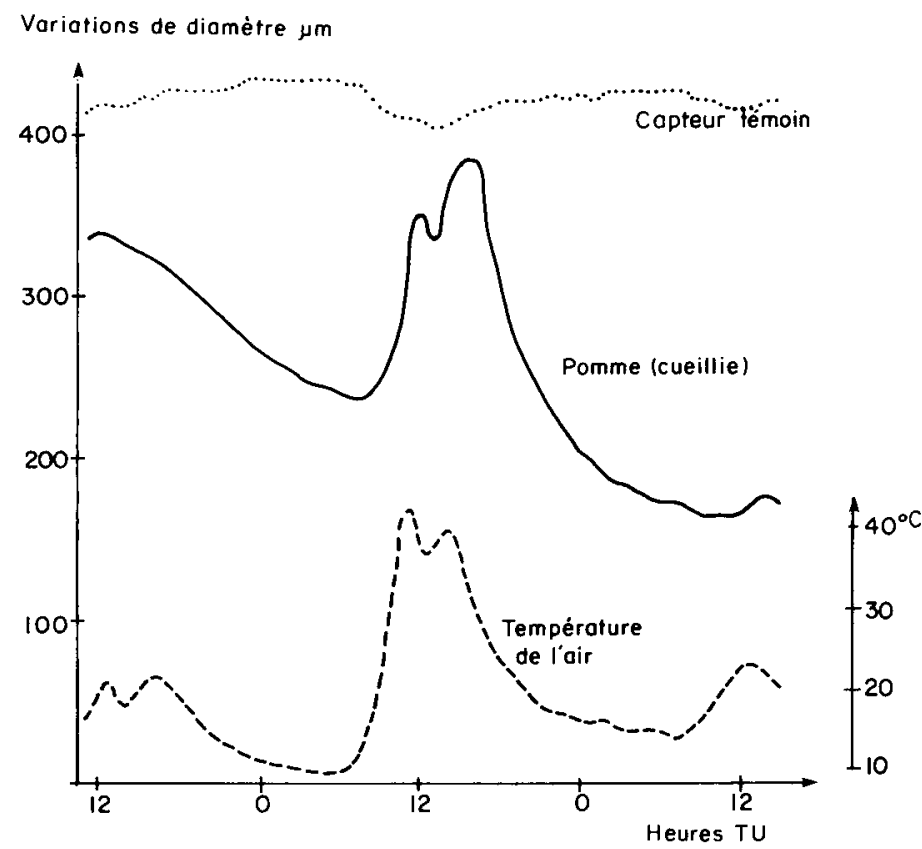

Figure 9

Dilatations thermiques d'une pomme séparée de l'arbre.

Expansion of a picked apple in relation to air temperature.

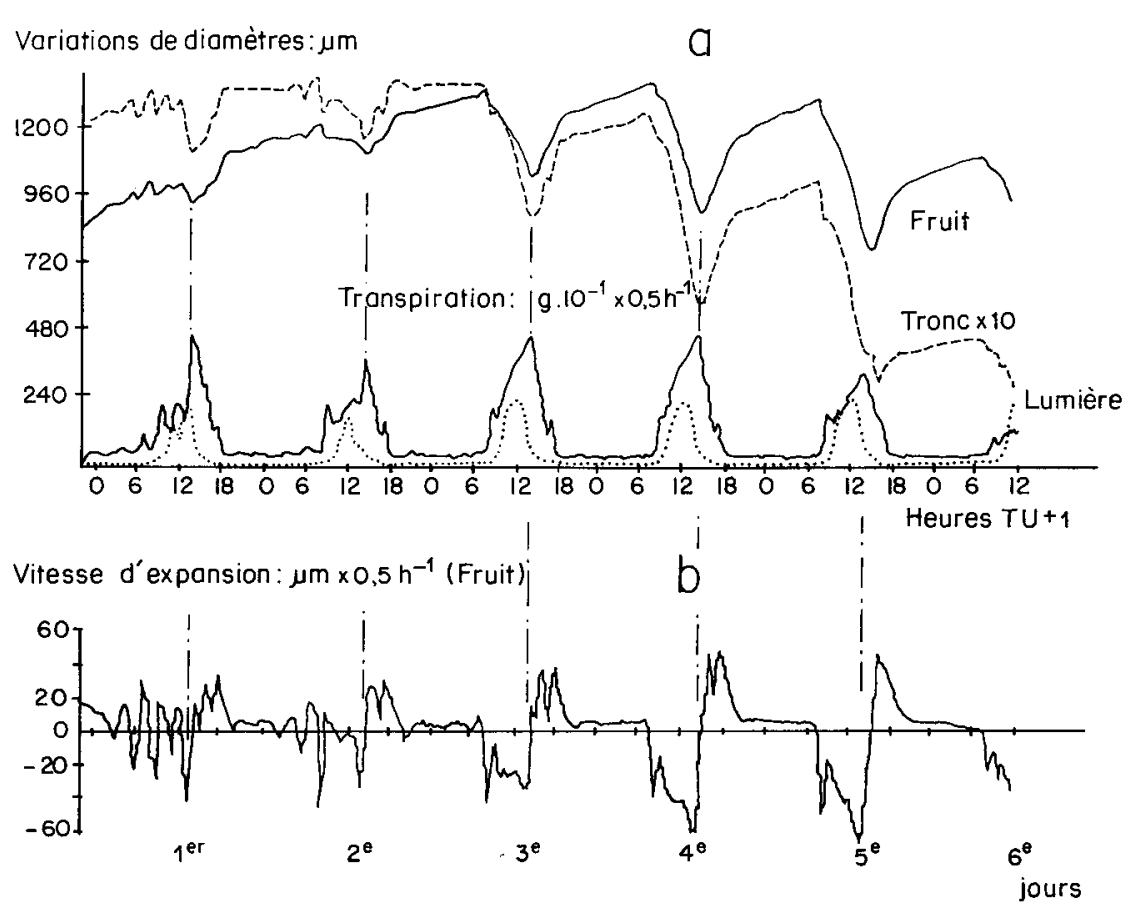

Figure 10

a) Evolutions, sur un arbre en pot, des diamètres du tronc et d'un citron en relation avec l'intensité de la transpiration et la luminosité.

b) Variation de la vitesse de contraction du citron.

d'autant plus forte que la transpiration est plus intense. Il faut noter qu'au lever du jour se place un épisode pendant lequel la transpiration et la contraction prennent, pour une courte durée, des valeurs anormalement fortes. Après le maximum de transpiration, les tissus réserves (fruit, tige) passent simultané- a) Variation in the diameter of stem and fruit of a potted lemon tree in relation to transpiration rate and light intensity.

b) Variation in the rate of contraction.

ment en expansion. Tout se passe comme si toute détente de la tension de transpiration (baisse de lumière par exemple) provoquait sans délai une inversion du flux hydrique au profit de la récupération des réserves, à condition que le sol fournisse assez d'eau. Ce phénomène a lieu en milieu d'après-midi par temps 
clair, mais également en pleine journée à l'occasion de passages nuageux ( $1^{\text {re }}$ journée).

Mais les effets de ces gradients de potentiel sur la mobilisation de l'eau des organes réservoirs dépendent aussi de la composition des tissus concernés. Ainsi, l'eau des pommes semble moins accessible que celle des parenchymes corticaux. Cette inaccessibilité relative paraît s'accentuer avec la maturation des fruits richesse en sucres).

On comprend alors la difficulté de relier une valeur absolue de la contraction d'un organe à une valeur absolue de potentiel hydrique (MOLZ \& KLEPPER, 1972).

\section{Rôle direct de la température}

Il semble logique de supposer qu'en plus de son rôle sur la transpiration, la température (température de l'air et température de surface), puisse avoir des effets directs sur les dimensions : en effet (fig. 9), la dilatation thermique du diamètre d'une pomme est d'environ $150 \mu \mathrm{m}$ pour une élévation de $20{ }^{\circ} \mathrm{C}$ (température air) sur une pomme de $70 \mathrm{~mm}$ de diamètre. Ceci paraît cohérent au regard du calcul sur une sphère fictive de même diamètre et composée d'eau pure dont la dilatation en diamètre serait d'environ $80 \mu \mathrm{m}$; le caractère composite du fruit (molécules organiques, gaz...) expliquant sa dilatation plus forte. Tronc et tiges paraissent beaucoup moins sensibles à ce phénomène (fig. 5).

\section{E. Décomposition de la variation globale de dimen- sion}

Au cours d'une période de $24 \mathrm{~h}$, les microvariations de diamètres observées sur les fruits ou les organes ligneux sont en fait le bilan des effets de 3 phénomènes principaux.

1. La croissance : augmentation de la taille ou du nombre des cellules grâce à l'arrivée d'eau et d'assimilats ; cette expansion cellulaire ne peut s'exprimer que lorsque le potentiel hydrique n'est pas trop bas, donc généralement en fin d'après-midi, la nuit et en début de matinée. Les nuits froides d'automne (températures inférieures à $8^{\circ} \mathrm{C}$ ) paraissent bloquer cette croissance chez le pommier.

Sur les tiges, la croissance secondaire en épaisseur peut, chez certaines espèces, être en pause au cours d'une partie de l'été.

2. Les échanges d'eau entre tissus : au cours de la journée, les contractions signalent les organes dont l'eau est mobilisée au profit de la transpiration des feuilles. L'amplitude de cette contraction est révélatrice d'une situation provisoirement négative du bilan en eau de la plante. En phase obscure, une partie de la dilatation observée peut s'interpréter comme une récupération de l'eau "avancée » au cours de la journée précédente.

3. Les variations thermiques: dilatation diurne, contraction nocturne en opposition avec les effets d'échanges d'eau entre organes.

Il est à remarquer que les variations d'origine thermique conduisent à sous-estimer la part des transferts d'eau dans les contractions diurnes et les dilatations nocturnes.
Suivant le moment de la saison, les circonstances climatiques et la disponibilité de l'eau dans le sol, il est tout à fait possible d'interpréter les évolutions globales de dimension par l'analyse des effets des 3 phénomènes principaux évoqués.

Dans le bilan des variations de dimension, il faut évoquer la respiration et la transpiration cuticulaire propres aux organes considérés, phénomènes dont les amplitudes doivent être négligeables par rapport aux précédents.

\section{DISCUSSION}

L'examen de la figure 10 peut servir de support à une interprétation globale des variations micrométriques de la taille d'un fruit et du tronc d'un citronnier en pot (25 l) en relation avec son état hydrique. Cette expérience a été menée en janvier dans une pièce à température pratiquement stable $\left(24 \pm 2^{\circ} \mathrm{C}\right)$, l'arbre étant placé devant une fenêtre exposée au Sud; les variations de poids de l'ensemble plante-pot étaient suivies en continu par pesée (balance de portée $60 \mathrm{~kg}$, sensibilité $0,1 \mathrm{~g}$ ) pour donner la vitesse de transpiration (surface du sol recouverte d'une feuille plastique). L'expérience a débuté en sol humide bien ressuyé et s'est entièrement déroulée sans nouvel apport d'eau. Les 4 dernières journées (de la $3^{\mathrm{e}}$ à la $6^{\mathrm{e}}$ ) ont bénéficié d'un temps clair et ensoleillé identique d'un jour à l'autre (courbe de luminosité) ; les 2 premières, par contre, ont été perturbées par du brouillard et des passages nuageux. Si l'on examine l'allure des variations cumulées de diamètres sur fruit et tronc (amplifiées d'un coefficient 10 ) au cours des 2 premières journées, la tendance globale est à la croisssance pour le fruit et à la stabilité pour le tronc tandis que les amplitudes maximum des contractions diurnes sont

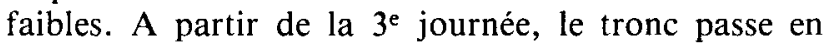
décroissance globale qui s'accélère jusqu'à la fin de l'expérience ; le fruit ralentit sa croissance nette (bilan sur $24 \mathrm{~h}$ ) pendant la $3^{\mathrm{e}}$ journée pour décroître nettement ensuite. Il faut noter, à partir de la $3^{\mathrm{e}}$ journée, l'augmentation des amplitudes de contractions diurnes qui se " creusent » d'autant plus que la plante « utilise » ses réserves hydriques pour compenser la baisse de disponibilité de l'eau dans le sol. Le procédé est efficace puisque jusqu'à la $4^{e}$ journée, le niveau maximum de transpiration (intensité) est maintenu. Par contre, malgré une sollicitation encore plus forte des réserves pendant la $5^{\mathrm{e}}$ journée, il y a une baisse de l'intensité maximum de transpiration qui dénote à la fois l'épuisement des ressources en eau du sol et des réserves ; apparemment, il n'y a pas encore eu de régulation stomatique liée à ce manque d'eau car la courbe de transpiration n'est pas tronquée, phénomène qui paraît se produire au cours de la $6^{\mathrm{e}}$ journée où la transpiration se met en palier bas dès le milieu de la matinée.

Notons que le suivi de plusieurs fruits du même arbre peut mettre en évidence des croissances nettes légèrement différentes, mais que tous les phénomènes liés aux variations de potentiels hydriques (maxi, mini, passages nuageux, etc...) sont d'un synchronisme parfait sur une même plante, synchronisme à l'image de celui observé entre le fruit et le tronc. Dans 
un terrain homogène, différents arbres d'une même parcelle réagissent avec le même "parallélisme ".

Tout semble se passer comme si, jusqu'à une certaine limite, l'utilisation des réserves hydriques d'une plante était tout à fait normale et permettait à celle-ci une stratégie de photosynthèse maximum qui tire partie de l'intensité lumineuse en compensant un déficit hydrique provisoire par une participation de ses réserves propres en eau, sans limiter son efficacité photosynthétique par fermeture stomatique.

Le phénomène de régulation stomatique n'interviendrait alors que lorsqu'un déficit prolongé aurait " épuisé " le compartiment réserve hydrique de la plante (quantitativement, une estimation prudente de ces réserves conduit à les évaluer entre 5 et $20 \mathrm{~m}^{3}$ d'eau pour un verger adulte de pommiers avec une charge moyenne en fruits).

En milieu d'après-midi, la transpiration diminue pour devenir très faible dans la nuit (transpiration cuticulaire). Si le sol est suffisamment humide, le bilan hydrique redevient positif (fin de la contraction), la plante reconstitue ses réserves. Par contre, si le sol ne fournit plus suffisamment d'eau, cette restauration des réserves est incomplète si la demande climatique persiste; l'amplitude de la contraction diurne augmente, révélant une sollicitation de plus en plus intense des réserves propres. Il est donc possible, expérimentalement et par le calcul, de déterminer, pour une catégorie d'organe et une espèce, une valeur limite de la contraction diurne qui révèle le besoin d'irriguer. Sur la figure 10, les amplitudes des contractions de la $4^{\mathrm{e}}$ journée sont une approche expérimentale de ces valeurs limites (fruits ou tronc).

\section{Amplitude critique de contraction diurne}

En revenant au tableau 1, il apparaît que la sollicitation relative des réserves n'a jamais dépassé 10 p. 100 de l'épaisseur totale de l'écorce dans des circonstances d'humidité suffisante du sol; dans ces mêmes conditions de sol, l'expérience permet de repérer l'amplitude relative maximum dans des conditions de très forte demande climatique, valeur qui ne sera pas dépassée tant qu'une disponibilité suffisante de l'eau dans le sol assure une absorption de la journée et autorise la récupération nocturne des réserves entamées.

Cette valeur relative limite peut être transformée en amplitude absolue de contraction en fonction du diamètre réel de l'organe mesuré.

Cette valeur peut constituer le paramètre d'asservissement pour un pilotage automatique de l'irrigation, qui tienne compte à la fois de la disponibilité réelle de l'eau accessible à l'ensemble du système racinaire et de la transpiration réelle du végétal concerné.

Des expériences exploratoires sur les tiges d'espèces annuelles (tiges de maïs et de tournesol) ont révélé des comportements strictement analogues qui laissent supposer que les hypothèses avancées sont largement généralisables.

\section{CONCLUSION}

Le suivi micrométrique de la dimension des fruits ou des tiges paraît un outil performant d'accès au bilan hydrique instantané d'une plante. Mais l'interprétation des contractions et dilatations observées doit tenir compte de l'ensemble des phénomènes principaux en jeu (transfert d'assimilats, échange d'eau, dilatations thermiques) examinés à la lumière des gradients de potentiels hydriques dans l'ensemble du système sol, rhizosphère, plante, atmosphère.

La signification des mesures paraît déjà suffisamment claire pour envisager des applications pratiques dans le domaine du pilotage de l'irrigation à travers les propres réactions des plantes aux modifications de leur milieu (Brevet INRA n ${ }^{\circ} 84.020 .20^{*}$ ).

\section{Reçu le 5 décembre 1984 Accepté le 9 avril 1985.}

\footnotetext{
* Brevet déposé le 9 février 1984 au nom de l'INRA : «Procédé et dispositif de commande automatique de l'irrigation de végétaux ».
}

\section{RÉFÉRENCES BIBLIOGRAPHIQUES}

Bartholomew E. T., 1923. Internal decline of lemons. III. Water deficits in lemon fruit caused by excessive leaf evaporation. $A n n . J$. Bot., 13, $102-117$.

Chaney W. R., Kozlowski T. T., 1971. Water transport in relation to expansion or contraction of leaves and fruits of Calamondin orange. J. Hortic. Sci., 46, 71-81.

Halma F. F., 1934. Some phases in water relation in citrus. Proc. Am. Soc. Hortic. Sci., 31, 108-109.

Hodgson R. W., 1917. Some abnormal water relations in citrus trees of the arid South-West and their possible significance. Univ. Calif. Publ. Agric. Sci., 3, 37-55.

Katerji N. B., 1982. Etude et modélisation des transferts hydriques dans le système sol-plante-atmosphère. Thèse Doct. Etat, juin 1982, Paris VII, 197 p. + annexe, 97 p.
Lassoie J.-P., 1973. Diurnal dimensional fluctuations in a Douglasfir stem in response to tree water status. For Sci., 19, 251-255.

Molz F. J., Klepper B., 1972. Radial propagation of water potentials in stems. Agron. J., 65, 469-473.

Powell D. B. B., Thorpe M. R., 1975. Dynamic aspects of plant water relations. 5th Long Ashton Symposium, 12-17 April 1975: " Environmental effects on crops physiology », 28 p.

Scholander P. F., Hannel H. T., Bradstreet E. D., Hemmingsen E. A., 1965. Sap pressure in vascular plants. Science, 148, 339346.

Schroeder C. A., Wiedland P. A., 1956. Diurnal fluctuation in size in various parts of the avocado tree and fruit. Proc. Am. Soc: Hortic. Sci., 68, 253-358. 